\title{
Atlanto-Axial Subluxation of the Cervical Spine As the Initial Presentation of Rheumatoid Arthritis : A Case Report
}

\author{
Mishra $\mathrm{Dk}^{1}$,Singhal $\mathrm{S}^{2}$, Manchanda $\mathrm{B}^{3}$,
}

\begin{abstract}
Rheumatoid arthritis (RA) is a chronic systemic inflammatory disease affecting a significant percentage of the population. Rheumatoid arthritis synovitis affects mostly small hand and feet joints, although it may compromise any joint with a synovial lining. Although cervical involvement occurs usually in longstanding disease in over half of these patients, it is usually preceeded by findings in other joints, such as small joints of the hand. Clinical presentation in spine involvement can be variable, and symptoms may be due to neck pain or compressive myeloradiculopathy.

We report the case of a 62 year old female who presented to the medicine outpatient department with a 3 year history of disabling neck pain and episodes of paresthesias along with muscle weakness.

On laboratorial workup, positive rheumatoid factor(qualitative and quantitative) along and anti-citrullinated peptide antibody were found. Cervical spine xrays and magnetic resonance series revealed subluxation of the atlanto-axial joint, multiple erosions and odontoid process inflammatory pannus. A diagnosis of rheumatoid arthritis with primary and isolated cervical spine involvement as the initial finding was made and the patient was started on methotrexate and methylprednisolone and referred to the Dept. Of Neurosurgery for cervical spine fixation and fusion.
\end{abstract}

Keywords: Rheumatoid Arthritis; Cervical Spine; Cervical Myelopathy; Atlanto-axial subluxation

\section{Introduction}

Rheumatoid arthritis (RA) is a chronic systemic inflammatory disease that primarily affects bones, synovial joints, and ligaments but can also involve nearly every organ system. RA primarily affects the peripheral joints; however it can also have profound systemic effects on the cardiovascular, pulmonary, and hematologic systems $\left[^{\underline{1}-\underline{3}}\right]$. RA affects an estimated $1-2 \%$ of the world's adult population. Although it is known to cause progressive joint destruction and severe disability, the current available disease-modifying antirheumatic drugs (DMARD's) have changed RA's natural history and reduced it's personal,social and economic burden. In addition to RA poorly understood etiology and pathogenesis, there is also heterogeneity in its clinical presentation. The main feature is synovitis, which can affect potentially any synovial membrane in the body (articular and periarticular).

The classic presentation is symmetric, additive and persistent inflammatory polyarthritis involving the proximal interphalangeal and metacarpophalangeal joints, wrists, elbows, shoulders, hips, knees, ankles and metatarsophalangeal joints. There can be also prolonged morning stiffness and functional impairment. The development of these symptoms is usually gradual but it can also be abrupt. Less frequently, initial RA involvement may be monoarticular or oligoarticular ${ }^{1}$. Spine involvement is a common late finding and it's mostly limited to the cervical spine. It occurs in over half the patients after an average of 10-year of disease duration $^{2}$. The authors report a case of isolated cervical spine involvement as the initial manifestation of RA.

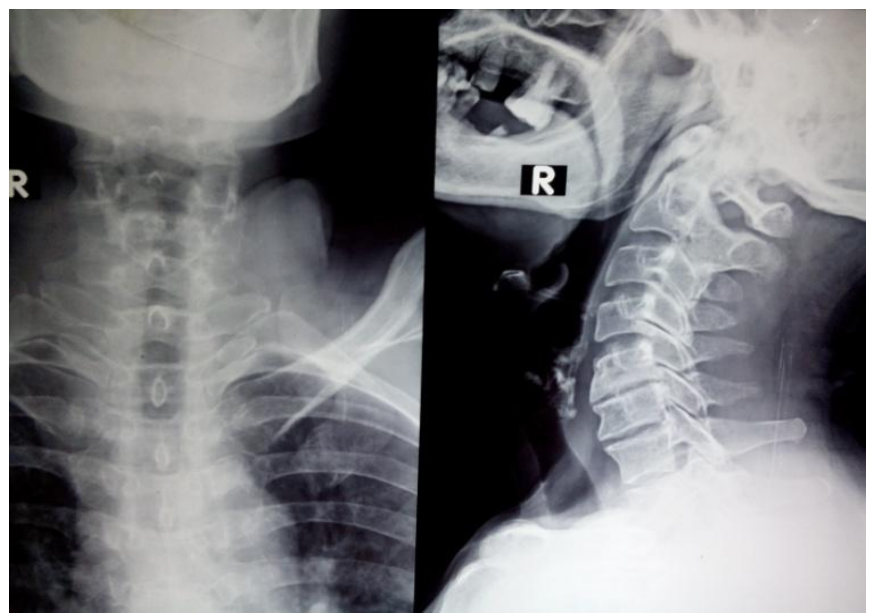

A full flexion xray of the cervical spine clearly showing showing atlantoaxial subluxation. 
Atlanto-Axial Subluxation of the Cervical Spine As the Initial Presentation of Rheumatoid ...

\section{Clinical Case}

A 62 year old female presented to the medicine outpatient clinic for complaints of a 3 year history of disabling neck pain, episodes of paresthesias and muscle weakness. The pain started 3 years ago and gradually progressed over the course of time. No triggering event or traumatic event was recorded. The pain was associated with a history of tingling and numbness originating at the back of the neck which was gradually increasing in intensity and was now severe enough to interfere with activities of daily living. During the entire period, the patient was treated multiple times with oral and intramuscular non-steroidal anti-inflammatory drugs, with only temporary relief. The patient had no significant past medical or surgical history and no history of tobacco or alcohol use was reported.

The physical examination revealed normal blood pressure, pulse and respiratory rate with a normal oral temperature. Chest and abdomen extremities revealed no abnormalities. There was pain and tenderness during palpation of the cervical spine along with loss of sensations over the nape of the neck. Severe limitation of the cervical spine movement ( maximum of 40 degrees rotation and 10 degrees of lateral flexion) was present. No evidence of cranial nerve dysfunction, pyramidal signs or signs of respiratory distress were found.

Laboratory workup revealed a normal complete blood count and normal kidney and liver function tests. C-reactive protein levels were elevated<insert value> and ESR was $54 \mathrm{~mm}$ in the first hour. Immunology tests revealed a positive rheumatoid factor and positive titer of anti-citrullinated peptide antibody. The Chest xray was unremarkable. In view of symptoms and examination, a cervical spine anterior and lateral xray was performed which showed atlanto-axial subluxation. In view of these findings, an Magnetic resonance imaging of the entire cervical spine was performed which revealed classical atlanto-axial subluxation, multiple erosions and odontoid process inflammatory pannus.

On the basis of these investigations, Rheumatoid arthritis of the cervical spine was diagnosed and the patient was started on methotrexate ( $15 \mathrm{mg} /$ week), prednisone $(20 \mathrm{mg}$ tds), folic acid(10mg per week) along with acelofenac (100mg SOS) and the patient was referred to neurosurgery for cervical spine fixation and fusion.
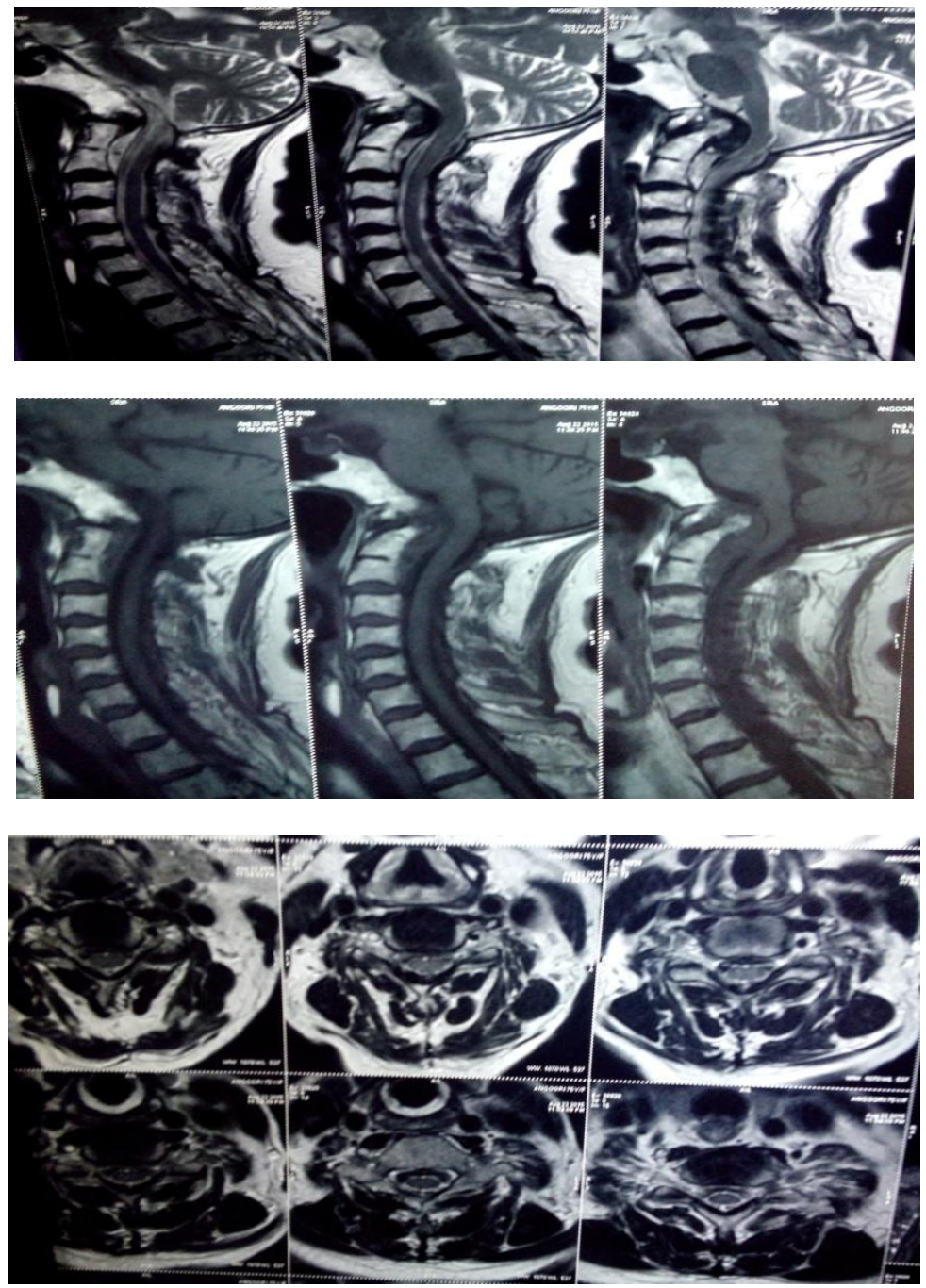
T1(left) and T2(right) weighted images showing atlantoaxial subluxation, erosions of the odontoid process and pannus inflammation.

\section{Discussion}

The authors report this case because, although cervical spine involvement is a common feature in longstanding disease, it is a very unusual form of primary presentation of RA. Axial skeleton involvement is a common feature of RA. It has a variable prevalence ranging from 25 to $80 \%$, with the cervical spine being almost exclusively affected. Although typical of longstanding disease, it may develop rather early with a reported prevalence of $10 \%$ after 2 years of disease duration and being increasingly frequent with ongoing inflammatory activity ${ }^{4}$. Although cervical subluxations are common, they are usually not seen as the initial or presenting feature in patients with rheumatoid arthritis. Their clinical presentation is often asymptomatic or associated with neck pain. Neurological complications are less frequent and tend to occur later in the disease course .

There are three types of cervical spine involvement in rheumatoid arthritis(RA). The most common is AAS (49\%), followed by superior migration of the odontoid (SMO, 38\%) and subaxialsubluxation (SAS, 10$20 \%$ ). In AAS, synovitis of the transverse ligament causes laxity and erosions of the odontoid processin which case the anterior arch of the atlas may move forward on neck flexion and upper cervical cord compression may occur $^{1}$. SMO is due to the erosion and bone lossin the occipitoatlantal and atlantoaxial joints, which causes the cranial migration of the odontoid process and compression of vitalstructures of the brainstem. In SAS there is unstable movement of vertebrae below the axis due to destruction of facets, intervertebral discs and interspinous ligaments $^{1}$. The earliest and most frequent symptom of cervical involvement is neck pain radiating to the occiput $^{8}$. Other symptoms may include a sensation of head falling forward upon flexion, dysphagia, vertigo, dysarthria, nystagmus, peripheral paresthesias, quadriparesis, loss of sphincter control, respiratory dysfunction and consciousness changes ${ }^{9}$. Transient ischemic attacks may occur due to vertebral artery compromise. Suggestive physical findings include loss of physiological lordosis, painful and limited active and passive cervical movements. Diagnostic confirmation is radiological and should combine the use of cervical lateral view plain radiography in full flexion (to assess the AAS) and cervical MRI (to assess synovitis and neural structures compression $)^{4}$. In lateral plain radiography, the distance between the posterior margin of the anterior ring of the atlas and the anterior surface of the odontoid process (anterior atlantodental distance) is considered abnormal when $>3 \mathrm{~mm}$ in the adult patient with $\mathrm{RA}^{10}$. Although an anterior atlantodental distance of $\geq 9 \mathrm{~mm}$ was associated with an increased incidence of paralysis, a distance of $\leq 14 \mathrm{~mm}$ between the posterior surface of the odontoid and the anterior margin of the posterior ring of the atlas showed a much higher sensitivity and negative predictive value for poor neurologic outcomes ${ }^{11}$.

Treatment may be surgical or conservative, depending on the presence or absence of signs of spinal cord compression, respectively. DMARD's are the cornerstone of RA therapy and they are essential for controlling inflammation and preventing joint damage in both peripheral and axial joints. There is some evidence that DMARD combination therapy may offer benefit over monotherapy in preventing cervical spine involvement ${ }^{12}$. Conservative treatment included the use of stiff collars for stabilization but their benefit in preventing serious injury is still controversial; its use should be decided on an individual basis ${ }^{12,13}$. The role and safety of cervical physical exercises is also uncertain. Patients with refractory pain or evidence of spinal cord compromise must be submitted to surgery in order to prevent irreversible neurological damage and death. Surgical management includes reduction and stabilization of the injured spine segment and decompression of neural structures ${ }^{14}$. Cervical involvement is a rather common late finding in RA and should always be considered when patients with long-standing disease present with new-onset cervical pain or neurologic symptoms. The reported case of cervical arthritis, as a presenting feature of RA, corroborates that this is a heterogeneous disease and may have atypical presentations for which the physician must be aware.

\section{References}

[1]. Brasington Junior RD. Clinical features of rheumatoid arthritis. In: Hochberg MC, Silman AJ, Smolen JS, Weinblatt ME, Weisman MH. Rheumatology. Philadelphia: Elsevier, 2011:829-838.

[2]. Rheumatoid Arthritis Clinical Presentation. http://emedicine.medscape.com/article/331715-clinical\#aw2aab6b3b2. Accessed February 2012.

[3]. Rajangam K, Thomas IM. Frequency of cervical spine involvement in rheumatoid arthritis. J Indian Med Assoc 1995;93:138- -139.

[4]. Kauppi MJ, Barcelos A, da Silva JAP. Cervical complications of rheumatoid arthritis. Ann Rheum Dis 2005;64:355-358.

[5]. Haeusler U, Dybowski F, Wittkaemper TA, Kisters K, Godolias G, Braun J. Arthritis of the atlanto-axial joint with inflammatory neck pain as a primary manifestation of seronegative rheumatoid arthritis. Dtsch Med Wochenschr 2010;135:1729-1732.

[6]. Jiménez Caballero PE, Ayuga Loro F, Muñoz Escudero F, Lobato Casado P. Cervical myelopathy by rheumatoid pannus as onset form ofrheumatoid arthritis. Med Clin (Barc) 2010;135:432- -433.

[7]. Morizono Y, Sakou T, Kawaida H. Upper cervical involvement in rheumatoid arthritis. Spine 1987;12:721-725.

[8]. StevensJC, Cartlidge NE, Saunders M, Appleby A, Hall M, Shaw DA. Atlanto-axialsubluxation and cervical myelopathy in rheumatoid arthritis. Q J Med 1971;40:391.

[9]. Mayer JW, Messner RP, Kaplan RJ. Brain stem compression in rheumatoid arthritis. JAMA 1976;236:2094. 
[10]. Nakano KK, Schoene WC, Baker RA, Dawson DM. The cervical myelopathy associated with rheumatoid arthritis: analysis of patients, with 2 postmortem cases. Ann Neurol 1978;3:144.

[11]. Boden SD, Dodge LD, Bohlman HH, Rechtine GR. Rheumatoid arthritis of the cervical spine. A long-term analysis with predictors of paralysis and recovery. J Bone Joint Surg Am 1993; 75:1282-1297.

[12]. Neva MH, Kauppi MJ, Kautiainen $\mathrm{H}$, et al. Combination drug therapy retardsthe development of rheumatoid atlantoaxialsubluxations. Arthritis Rheum 2000;43:2397.

[13]. Kauppi MJ, Anttila P. A stiff collar can restrict atlantoaxial instability in rheumatoid cervical spine in selected cases. Ann Rheum Dis 1995; 54:305-307.

[14]. Althoff B, Goldie IF. Cervical collarsin rheumatoid atlanto-axial subluxation: a radiographic comparison. Ann Rheum Dis 1980;39:485.

[15]. Filipe Araújo, Inês Silva Cervical spine involvement as initial manifestation of rheumatoid arthritis: a case report Acta Reumatol Port. 2015;40:64-67 